\title{
Global Exponential Stability of Learning-Based Fuzzy Networks on Time Scales
}

\author{
Juan Chen, Zhenkun Huang, and Jinxiang Cai \\ School of Sciences, Jimei University, Xiamen 361021, China \\ Correspondence should be addressed to Zhenkun Huang; hzk974226@jmu.edu.cn
}

Received 17 June 2014; Accepted 14 August 2014

Academic Editor: Hui-Sheng Ding

Copyright (C) 2015 Juan Chen et al. This is an open access article distributed under the Creative Commons Attribution License, which permits unrestricted use, distribution, and reproduction in any medium, provided the original work is properly cited.

We investigate a class of fuzzy neural networks with Hebbian-type unsupervised learning on time scales. By using Lyapunov functional method, some new sufficient conditions are derived to ensure learning dynamics and exponential stability of fuzzy networks on time scales. Our results are general and can include continuous-time learning-based fuzzy networks and corresponding discrete-time analogues. Moreover, our results reveal some new learning behavior of fuzzy synapses on time scales which are seldom discussed in the literature.

\section{Introduction}

It is well known that many applications of neural networks exist in diverse areas such as optimization, signal and image processing, pattern recognition, and control system. These applications are based on stability of equilibrium points of the network models. Hence, stability criteria of equilibrium points of networks have been greatly investigated in the literature [1-4]. Meanwhile, more recently, there have been several publications on the theme of neural networks where fuzzy logic is used. Yang and Yang [2,5] and Yang et al. [6] have proposed a fuzzy cellular neural network to include and analyze the ambiguity or vagueness inherent in the inputs and outputs of neural networks. Further analysis of this type of networks can be found in the works of Yuan et al. [4], Liu and Tang [7], Huang and Zhang [8], Huang [9, 10], Chen and Liao [11], and the references therein.

It is welknown that the theory of time scales has a tremendous potential for applications in some mathematical models of real processes and phenomena studied in physics, population dynamics, biotechnology, economics, and so on. Meanwhile, it is unsuitable to study the stability for continuous and discrete system, respectively. Therefore, it is meaningful to study that on time scales which can unify the continuous and discrete situations. Many authors incorporate time scales into stability analysis of neural network models; we can refer to [12-15].

Stimulated by [16], we consider a class of networks of somatically crisp neurons with fuzzy learnable synapses on time scales $\mathbb{T}$ described by

$$
\begin{aligned}
u_{i}^{\Delta}(t)= & -a_{i} u_{i}(t)+\sum_{j=1}^{n} a_{i j} f_{j}\left(u_{j}(t)\right)+B_{i} \sum_{j=1}^{n} m_{i j}(t) p_{j} \\
& +b_{i} \bigvee_{j=1}^{n} b_{i j} f_{j}\left(u_{j}\left(t-\tau_{j}\right)\right) \\
& +c_{i} \bigwedge_{j=1}^{n} c_{i j} f_{j}\left(u_{j}\left(t-\tau_{j}\right)\right)+J_{i}, \\
m_{i j}^{\Delta}(t)= & -\alpha_{i} m_{i j}(t) \\
& +\left[\beta_{i} f_{i}\left(u_{i}(t)\right)+\gamma_{i} \bigwedge_{j=1}^{n} \gamma_{i j} f_{j}\left(u_{j}\left(t-\sigma_{j}\right)\right)\right. \\
& \left.+\delta_{i} \bigvee_{j=1}^{n} \delta_{i j} f_{j}\left(u_{j}\left(t-\sigma_{j}\right)\right)\right] p_{j},
\end{aligned}
$$


where $i \in \mathbb{N}=\{1,2, \ldots, n\}, u_{i}(t)$ denotes the state of neuron $i$ at time $t, a_{i}$ denotes the passive negative stabilizing feedback of neuron $i, a_{i j}, b_{i j}, c_{i j}, \gamma_{i j}$, and $\delta_{i j}$ denote the synaptic weights of the various fuzzy and nonfuzzy synapses of neuron $i, B_{i}, b_{i}, c_{i}, \beta_{i}, \gamma_{i}, \delta_{i}$ are disposable constants, and $m_{i j}$ denotes a learnable synaptic weight of neuron when it is presented with a constant input signal vector $p=\left(p_{1}, p_{2}, \ldots, p_{n}\right)$; the external bias to the network is denoted by the constant vector $J=\left(J_{1}, J_{2}, \ldots, J_{n}\right)$. The operators $\bigvee$ and $\bigwedge$ denote, respectively, the "max" and "min" operators used in fuzzy logic. The learning equation is based on the Hebbian-type $[16,17]$ unsupervised algorithm modified by the introduction of a forgetting term as proposed by Amari [18]. By using auxiliary variables $v_{i}(t)=\sum_{j=1}^{n} m_{i j}(t) p_{i}$, one gets

$$
\begin{aligned}
& u_{i}^{\Delta}(t)=-a_{i} u_{i}(t)+\sum_{j=1}^{n} a_{i j} f_{j}\left(u_{j}(t)\right)+B_{i} v_{i}(t) \\
& +b_{i} \bigvee_{j=1}^{n} b_{i j} f_{j}\left(u_{j}\left(t-\tau_{j}\right)\right) \\
& +c_{i} \bigwedge_{j=1}^{n} c_{i j} f_{j}\left(u_{j}\left(t-\tau_{j}\right)\right)+J_{i} \\
& v_{i}^{\Delta}(t)=-\alpha_{i} v_{i}(t) \\
& +\left[\beta_{i} f_{i}\left(u_{i}(t)\right)+\gamma_{i} \bigwedge_{j=1}^{n} \gamma_{i j} f_{j}\left(u_{j}\left(t-\sigma_{j}\right)\right)\right. \\
& \left.+\delta_{i} \bigvee_{j=1}^{n} \delta_{i j} f_{j}\left(u_{j}\left(t-\sigma_{j}\right)\right)\right] c \\
& i=1,2, \ldots, n, t>0,
\end{aligned}
$$

where $c=\sum_{j=1}^{n} p_{j}^{2}, i \in \mathbb{N}=\{1,2, \ldots, n\}, t \in \mathbb{T}^{+}$. Equation (2) is quite general and it includes several well known neural networks [16] and its difference analogue is

$$
\begin{aligned}
& \Delta u_{i}(m)=-a_{i} u_{i}(m)+\sum_{j=1}^{n} a_{i j} f_{j}\left(u_{j}(m)\right)+B_{i} v_{i}(m) \\
& +b_{i} \bigvee_{j=1}^{n} b_{i j} f_{j}\left(u_{j}\left(m-\tau_{j}\right)\right) \\
& +c_{i} \bigwedge_{j=1}^{n} c_{i j} f_{j}\left(u_{j}\left(m-\tau_{j}\right)\right)+J_{i}, \\
& \Delta v_{i}(m)=-\alpha_{i} v_{i}(m) \\
& +\left[\beta_{i} f_{i}\left(u_{i}(m)\right)+\gamma_{i} \bigwedge_{j=1}^{n} \gamma_{i j} f_{j}\left(u_{j}\left(m-\sigma_{j}\right)\right)\right. \\
& \left.+\delta_{i} \bigvee_{j=1}^{n} \delta_{i j} f_{j}\left(u_{j}\left(m-\sigma_{j}\right)\right)\right] c
\end{aligned}
$$

where $\Delta u_{i}(m)=u_{i}(m+1)-u_{i}(m)$ and $\Delta v_{i}(m)=v_{i}(m+1)-$ $v_{i}(m)$ are the forward difference operators and $m, \tau_{j}, \sigma_{j} \in \mathbb{Z}$, $i \in \mathbb{N}$.

For convenience, we let $b_{i j}^{\ell}:=b_{i} b_{i j}, c_{i j}^{\ell}:=c_{i} c_{i j}, \gamma_{i j}^{\ell}:=\gamma_{i} \gamma_{i j}$, $\delta_{i j}^{\ell}:=\delta_{i} \delta_{i j}$, and $c=1$. Then, $(2)$ reduces to

$$
\begin{aligned}
u_{i}^{\Delta}(t)= & -a_{i} u_{i}(t)+\sum_{j=1}^{n} a_{i j} f_{j}\left(u_{j}(t)\right)+\bigvee_{j=1}^{n} b_{i j}^{\ell} f_{j}\left(u_{j}\left(t-\tau_{j}\right)\right) \\
& +\bigwedge_{j=1}^{n} c_{i j}^{\ell} f_{j}\left(u_{j}\left(t-\tau_{j}\right)\right)+B_{i} v_{i}(t)+J_{i}, \\
v_{i}^{\Delta}(t)= & -\alpha_{i} v_{i}(t)+\beta_{i} f_{i}\left(u_{i}(t)\right)+\bigwedge_{j=1}^{n} \gamma_{i j}^{\ell} f_{j}\left(u_{j}\left(t-\sigma_{j}\right)\right) \\
& +\bigvee_{j=1}^{n} \delta_{i j}^{\ell} f_{j}\left(u_{j}\left(t-\sigma_{j}\right)\right) .
\end{aligned}
$$

Correspondingly, synaptic dynamic equation is as follows:

$$
\begin{aligned}
m_{i j}^{\Delta}(t)= & -\alpha_{i} m_{i j}(t) \\
& +\left[\beta_{i} f_{i}\left(u_{i}(t)\right)+\bigwedge_{j=1}^{n} \gamma_{i j}^{\ell} f_{j}\left(u_{j}\left(t-\sigma_{j}\right)\right)\right. \\
& \left.+\bigvee_{j=1}^{n} \delta_{i j}^{\ell} f_{j}\left(u_{j}\left(t-\sigma_{j}\right)\right)\right] p_{j},
\end{aligned}
$$

where $\sum_{j=1}^{n} p_{j}^{2}=1$. In this paper, we will study learningbased fuzzy networks (4) on time scales. Without the learning component and $\mathbb{T}=\mathbb{R}$, (4) will include fuzzy networks discussed by several authors recently (see [2, 4, 7, 9-11]). In the absence of fuzzy synapses, our model reduces to the most commonly studied Hopfield-type neural network. Moreover, by using the calculus theory on time scale to unify and generalize discrete-time and continuous-time learning-based fuzzy networks, we can establish new sufficient conditions to ensure existence and global exponential stability of equilibrium of (4).

The paper is organized as follows. In Section 2, we present some basic definitions concerning the calculus on time scales. In Section 3, we develop Lyapunov functions technique on time scale to give some sufficient conditions of global exponential stability for (4). In Section 4, an example is given to illustrate the effectiveness of our main results. Conclusions remarks are given in Section 5.

\section{Preliminaries on Time Scales}

The basic calculus theory on time scales was initiated by Hilger [19, 20], and Agarwal et al. summarize and organize much of relative results in monograph [21-23]. In this section, we will introduce some basic definitions and lemmas. 
Definition 1. A time scale $\mathbb{T}$ is arbitrary nonempty closed subset of the real set $\mathbb{R}$ with the topology and ordering inherited from $\mathbb{R}$.

Definition 2. On any time scale $\mathbb{T}$, one defines the forward and backward jump operators by $\sigma(t):=\inf \{s \in \mathbb{T}: s>t\}$ and $\rho(t):=\sup \{s \in \mathbb{T}: s<t\}$; one puts inf $\emptyset:=\sup \mathbb{T}$ and $\sup \emptyset:=\inf \mathbb{T}$, where $\emptyset$ denotes the empty set. A point $t$ is said to be left-dense if $t>\inf \mathbb{T}$ and $\rho(t)=t$, right-dense if $t<\sup \mathbb{T}$ and $\sigma(t)=t$, left-scattered if $\rho(t)<t$, and rightscattered if $\sigma(t)>t$. The graininess function $\mu$ for a time scale $\mathbb{T}$ is defined by $\mu(t):=\sigma(t)-t$. If $\mathbb{T}$ has a left-scattered maximum $m$, then one defined $\mathbb{T}^{k}$ to be $\mathbb{T}-m$. Otherwise, $\mathbb{T}^{k}=\mathbb{T}$.

Definition 3. For a function $f: \mathbb{T} \rightarrow \mathbb{R}$ (the range $\mathbb{R}$ of $f$ may be actually replaced by Banach space), the (delta) derivative is defined by

$$
f^{\Delta}=\frac{f(\sigma(t))-f(t)}{\sigma(t)-t},
$$

if $f$ is continuous at $t$ and $t$ is right-scattered. If $t$ is not rightscattered then the derivative is defined by

$$
f^{\Delta}=\lim _{s \rightarrow t} \frac{f(t)-f(s)}{t-s}
$$

provided this limit exists.

Definition 4. A function $F: \mathbb{T}^{k} \rightarrow \mathbb{R}$ is called a deltaantiderivative of $f: \mathbb{T} \rightarrow \mathbb{R}$ provided $F^{\Delta}=f$ holds for all $t \in \mathbb{T}^{k}$. In this case, one defines the integral of $f$ by

$$
\int_{a}^{t} f(s) \Delta s=F(t)-F(a) \quad \forall t \in \mathbb{T},
$$

and one has the following formula:

$$
\int_{t}^{\sigma(t)} f(s) \Delta s=\mu(t) f(t) \quad \forall t \in \mathbb{T}^{k} .
$$

Definition 5. A function $f: \mathbb{T} \rightarrow \mathbb{R}$ is called right-dense continuous provided it is continuous at right-dense points of $\mathbb{T}$ and the left sided limit exists (finite) at left-dense point of $\mathbb{T}$. The set of all right-dense continuous functions on $\mathbb{T}$ is defined by $C_{\text {rd }}=C_{\text {rd }}(\mathbb{T})=C_{\text {rd }}(\mathbb{T}, \mathbb{R})$.

Definition 6. One says that a function $p: \mathbb{T} \rightarrow \mathbb{R}$ is regressive provided $1+\mu(t) p(t) \neq 0$ for all $t \in \mathbb{T}$. The set of all regressive functions on a time scale $\mathbb{T}$ forms an Abelian group under the addition $\oplus$ defined by $p \oplus q:=$ $p+q+\mu p q$. The additive inverse in this group is denoted by $\ominus p:=-p /(1+\mu p)$. One then defines subtraction $\ominus$ on the set of regressive functions by $p \ominus q:=p \oplus(\ominus q)$. It can be shown that $p \oplus(\ominus q)=-(p-q) /(1+\mu q)$. The set of all regressive and right-dense continuous functions will be denoted by $\mathscr{R}$.

Definition 7. One defines the set $\mathscr{R}^{+}$of all positively regressive elements of $\mathscr{R}$ by $\mathscr{R}^{+}=\{f \in \mathscr{R}: 1+\mu(t) f(t)>0$ for all $t \in \mathbb{T}\}$.
Next, we give the definition of the exponential function and list some of its properties.

Definition 8. If $p(t) \in \mathscr{R}$, one defines the generalized exponential function as

$$
\begin{aligned}
e_{p}(t, s) & =\exp \left(\int_{s}^{t} \xi_{\mu(\tau)}(p(\tau)) \Delta \tau\right) \\
& =\exp \left(\int_{s}^{t} \frac{\log (1+\mu(\tau) p(\tau))}{\mu(\tau)} \Delta \tau\right) \quad \text { for } t, \tau \in \mathbb{T},
\end{aligned}
$$

where $\xi_{h}(x)=\log (1+x h) / h, h>0$, and $\xi_{0}(x)=x$.

Remark 9. The exponential function $e_{p}\left(\cdot, t_{0}\right)$ is the unique solution of the IVP $x^{\Delta}=p(t) x, x\left(t_{0}\right)=1$ for $p(t) \in \mathscr{R}$. As $\mathbb{T}=\mathbb{R}, e_{p}\left(t, t_{0}\right)=\exp \left(\int_{t_{0}}^{t} p(s) d s\right)$.

Lemma 10. If $p, q \in \mathscr{R}$, then

(i) $e_{0}(t, s) \equiv 1$ and $e_{p}(t, t)=1$;

(ii) $e_{p}(\sigma(t), s)=(1+\mu(t) p(t)) e_{p}(t, s)$;

(iii) $e_{p}(t, s) e_{p}(s, r)=e_{p}(t, r)$;

(iv) $e_{p}(t, s)=1 / e_{p}(s, t)=e_{\ominus p}(s, t)$;

(v) $e_{p}(t, s)>0$, for $p \in \mathscr{R}^{+}$;

(vi) $e_{p}(t, s) e_{q}(t, s)=e_{p \oplus q}(t, s)$;

(vii) $e_{p}(t, s) / e_{q}(t, s)=e_{p \ominus q}(t, s)$.

Lemma 11 (see [3]). If $p \in \mathscr{R}$ and $a, b, c \in \mathbb{T}$, then

$$
\int_{a}^{b} p(t) e_{p}(c, \sigma(t)) \Delta t=e_{p}(c, a)-e_{p}(c, b) .
$$

If $-p \in \mathscr{R}^{+}$, then

$$
e_{-p}(t, s) \leq \exp \left\{-\int_{s}^{t} p(u) d u\right\}, \quad t \geq s .
$$

Lemma 12 (see [22]). Let $y, f \in C_{r d}$ and $p \in \mathscr{R}^{+}$. Then, $y^{\Delta}(t) \leq p(t) y(t)+f(t), t \in \mathbb{T}$, implies

$$
y(t) \leq y\left(t_{0}\right) e_{p}\left(t, t_{0}\right)+\int_{t_{0}}^{t} e_{p}(t, \sigma(\tau)) f(\tau) \Delta \tau, \quad t \in \mathbb{T} .
$$

Lemma 13 (see $[2,5]$ ). Suppose $x=\left(x_{1}, x_{2}, \ldots, x_{n}\right), y=$ $\left(y_{1}, y_{2}, \ldots, y_{n}\right)$ are any two vectors in $\mathbb{R}^{n}$ :

$$
\begin{aligned}
& \left|\bigwedge_{j=1}^{n} \alpha_{i j} f_{j}\left(x_{j}\right)-\bigwedge_{j=1}^{n} \alpha_{i j} f_{j}\left(y_{j}\right)\right| \leq \sum_{j=1}^{n}\left|\alpha_{i j}\right|\left|f_{j}\left(x_{j}\right)-f_{j}\left(y_{j}\right)\right|, \\
& \left|\bigvee_{j=1}^{n} \beta_{i j} f_{j}\left(x_{j}\right)-\bigvee_{j=1}^{n} \beta_{i j} f_{j}\left(y_{j}\right)\right| \leq \sum_{j=1}^{n}\left|\beta_{i j}\right|\left|f_{j}\left(x_{j}\right)-f_{j}\left(y_{j}\right)\right|,
\end{aligned}
$$

$\forall i \in \mathbb{N}$ 
Throughout this paper, we make the following basic assumptions:

$\left(\mathscr{A}_{1}\right)$ The functions $g_{i}, f_{j}(i, j \in \mathbb{N})$ are Lipschitz continuous on $\mathbb{R}$ with the Lipschitz constants $\widetilde{L_{i}}$ and $L_{j}$, respectively; that is, $\left|f_{j}(y)-f_{j}(x)\right| \leq L_{j}|y-x|$, $\left|g_{i}(y)-g_{i}(x)\right| \leq \widetilde{L_{i}}|y-x|$.

\section{Main Results}

In this section, we study the global exponential stability of the unique equilibrium for (4) on time scale by using Lyapunov method.

Theorem 14. Suppose that (4) satisfies $\left(\mathscr{A}_{1}\right)$; if there exist positive constants $\lambda_{i}, \xi_{i}$, and $p$ such that

$$
\begin{aligned}
& \left(\mathscr{A}_{2}\right) \\
& \lambda_{i} p+(1+\mu(t) p) \\
& \times\left[-2 \lambda_{i} a_{i}+\lambda_{i}\left(\sum_{j=1}^{n}\left(\left|a_{i j}\right|+\alpha_{i j}\right) L_{j}+\left|B_{i}\right|\right)\right. \\
& +\xi_{i}\left|\beta_{i}\right| L_{i}+\sum_{j=1}^{n} \lambda_{j}\left|a_{j i}\right| L_{i} \\
& +\mu(t)\left[\lambda_{i} a_{i}^{2}+\lambda_{i} a_{i}\left(\sum_{j=1}^{n}\left(\left|a_{i j}\right|+\alpha_{i j}\right) L_{j}+\left|B_{i}\right|\right)\right. \\
& +\xi_{i}\left|\beta_{i}\right| L_{i}\left(\left|\beta_{i}\right| L_{i}+\alpha_{i}+\sum_{j=1}^{n} \beta_{i j} L_{j}\right) \\
& +\sum_{j=1}^{n} \lambda_{j}\left|a_{j i}\right| L_{i} \\
& \left.\left.\times\left(n\left|a_{j i}\right| L_{i}+a_{j}+\alpha_{j i} L_{i}+\left|B_{j}\right|\right)\right]\right] \\
& +\sum_{j=1}^{n} \lambda_{j}\left(1+\mu\left(t+\tau_{i}\right) p\right) e_{p}\left(t+\tau_{i}, t\right) \\
& \times \alpha_{j i} L_{i}\left[1+\mu\left(t+\tau_{i}\right)\left(n \alpha_{j i} L_{i}+a_{j}+\left|a_{j i}\right| L_{i}+\left|B_{j}\right|\right)\right] \\
& +\sum_{j=1}^{n} \xi_{j}\left(1+\mu\left(t+\sigma_{i}\right) p\right) e_{p}\left(t+\sigma_{i}, t\right) \beta_{j i} L_{i} \\
& \times\left[1+\mu\left(t+\sigma_{i}\right)\left(n \beta_{j i} L_{i}+\alpha_{j}+\left|\beta_{j}\right| L_{j}\right)\right]<0,
\end{aligned}
$$

$$
\begin{aligned}
& \xi_{i} p+(1+\mu(t) p) \\
& \times\left[\lambda_{i}\left|B_{i}\right|-2 \xi_{i} \alpha_{i}+\xi_{i}\left|\beta_{i}\right| L_{i}+\xi_{i} \sum_{j=1}^{n} \beta_{i j} L_{j}\right. \\
&+\mu(t)\left[\lambda_{i}\left|B_{i}\right|\left(\left|B_{i}\right|+a_{i}+\sum_{j=1}^{n}\left(\left|a_{i j}\right|+\alpha_{i j}\right) L_{j}\right)\right. \\
&\left.\left.+\alpha_{i} \xi_{i}\left(\alpha_{i}+\left|\beta_{i}\right| L_{i}+\sum_{j=1}^{n} \beta_{i j} L_{j}\right)\right]\right]<0
\end{aligned}
$$

where $\left|b_{i j}^{\ell}\right|+\left|c_{i j}^{\ell}\right|=\alpha_{i j}$ and $\left|\gamma_{i j}^{\ell}\right|+\left|\delta_{i j}^{\ell}\right|=\beta_{i j}, i, j \in \mathbb{N}$, then there exists a unique equilibrium $\left(u^{*}, v^{*}\right)=\left(u_{1}^{*}, \ldots, u_{n}^{*}, v_{1}^{*}, \ldots, v_{n}^{*}\right)$ of (4) which is globally exponentially stable; that is, every solution $(u, v)=\left(u_{1}, \ldots, u_{n}, v_{1}, \ldots, v_{n}\right)$ of $(4)$ satisfies

$$
\begin{aligned}
& \sum_{i=1}^{n}\left(u_{i}(t)-u_{i}^{*}\right)^{2}+\sum_{i=1}^{n}\left(v_{i}(t)-v_{i}^{*}\right)^{2} \leq M e_{\ominus p}(t, 0) \Phi_{\theta} \\
& \quad \longrightarrow 0 \text { as } t \longrightarrow+\infty
\end{aligned}
$$

where $M>0, \theta=\max _{j \in \mathbb{N}}\left(\tau_{j}, \sigma_{j}\right)$, and

$$
\Phi_{\theta}:=\left[\sum_{i=1}^{n} \sup _{-\theta \leq s \leq 0}\left(u_{i}(s)-u_{i}^{*}\right)^{2}+\sum_{i=1}^{n} \sup _{-\theta \leq l \leq 0}\left(v_{i}(l)-v_{i}^{*}\right)^{2}\right] \text {. }
$$

Proof. Similar to the proof of [16], we can prove (4) possesses a unique equilibrium $\left(u^{*}, v^{*}\right)=\left(u_{1}^{*}, \ldots, u_{n}^{*}, v_{1}^{*}, \ldots, v_{n}^{*}\right)$. Let $w_{i}(t)=u_{i}(t)-u_{i}^{*}$ and let $\rho_{i}(t)=v_{i}(t)-v_{i}^{*}$; then, we can rewrite (4) into

$$
\begin{aligned}
w_{i}^{\Delta}(t) \leq & -a_{i} w_{i}(t)+\sum_{j=1}^{n}\left|a_{i j}\right| L_{j}\left|w_{j}(t)\right| \\
& +\sum_{j=1}^{n}\left|b_{i j}^{\ell}\right| L_{j}\left|w_{j}\left(t-\tau_{j}\right)\right| \\
& +\sum_{j=1}^{n}\left|c_{i j}^{\ell}\right| L_{j}\left|w_{j}\left(t-\tau_{j}\right)\right|+\left|B_{i}\right|\left|\rho_{i}(t)\right| \\
= & -a_{i} w_{i}(t)+\sum_{j=1}^{n}\left|a_{i j}\right| L_{j}\left|w_{j}(t)\right| \\
& +\sum_{j=1}^{n}\left(\left|b_{i j}^{\ell}\right|+\left|c_{i j}^{\ell}\right|\right) L_{j}\left|w_{j}\left(t-\tau_{j}\right)\right|+\left|B_{i}\right|\left|\rho_{i}(t)\right| \\
= & -a_{i} w_{i}(t)+\sum_{j=1}^{n}\left|a_{i j}\right| L_{j}\left|w_{j}(t)\right| \\
& +\sum_{j=1}^{n} \alpha_{i j} L_{j}\left|w_{j}\left(t-\tau_{j}\right)\right|+\left|B_{i}\right|\left|\rho_{i}(t)\right|,
\end{aligned}
$$




$$
\begin{aligned}
\rho_{i}^{\Delta}(t) \leq & -\alpha_{i} \rho_{i}(t)+\left|\beta_{i}\right| L_{i}\left|w_{i}(t)\right|+\sum_{j=1}^{n}\left|\gamma_{i j}^{\ell}\right| L_{j}\left|w_{j}\left(t-\sigma_{j}\right)\right| \\
& +\sum_{j=1}^{n}\left|\delta_{i j}^{\ell}\right| L_{j}\left|w_{j}\left(t-\sigma_{j}\right)\right| \\
= & \left|\beta_{i}\right| L_{i}\left|w_{i}(t)\right|+\sum_{j=1}^{n}\left(\left|\gamma_{i j}^{\ell}\right|+\left|\delta_{i j}^{\ell}\right|\right) L_{j}\left|w_{j}\left(t-\sigma_{j}\right)\right| \\
& -\alpha_{i} \rho_{i}(t) \\
= & \left|\beta_{i}\right| L_{i}\left|w_{i}(t)\right|+\sum_{j=1}^{n} \beta_{i j} L_{j}\left|w_{j}\left(t-\sigma_{j}\right)\right|-\alpha_{i} \rho_{i}(t) .
\end{aligned}
$$

Now, we construct the Lyapunov function $F(t)=F_{1}(t)+$ $F_{2}(t)+F_{3}(t)+F_{4}(t)$, where

$$
\begin{aligned}
& F_{1}(t)=\sum_{i=1}^{n} \lambda_{i} w_{i}^{2}(t) e_{p}(t, 0), \\
& F_{2}(t)=\sum_{i=1}^{n} \xi_{i} \rho_{i}^{2}(t) e_{p}(t, 0) \\
& F_{3}(t)=\sum_{i=1}^{n} \sum_{j=1}^{n} \lambda_{i} \int_{t-\tau_{j}}^{t}\left\{\alpha_{i j} L_{j}\right. \\
& \times\left[1+\mu\left(s+\tau_{j}\right)\right. \\
& \left.\left.\times\left(n \alpha_{i j} L_{j}+a_{i}+\left|a_{i j}\right| L_{j}+\left|B_{i}\right|\right)\right]\right\} \\
& \times\left(1+\mu\left(s+\tau_{j}\right) p\right) e_{p}\left(s+\tau_{j}, 0\right) w_{j}^{2}(s) \Delta s \\
& F_{4}(t)=\sum_{i=1}^{n} \sum_{j=1}^{n} \xi_{i} \int_{t-\sigma_{j}}^{t}\left\{\beta _ { i j } L _ { j } \left[1+\mu\left(s+\sigma_{j}\right)\right.\right. \\
& \left.\left.\times\left(n \beta_{i j} L_{j}+\alpha_{i}+\left|\beta_{i}\right| L_{i}\right)\right]\right\} \\
& \times\left(1+\mu\left(s+\sigma_{j}\right) p\right) e_{p}\left(s+\sigma_{j}, 0\right) w_{j}^{2}(s) \Delta s \\
& F_{1}^{\Delta}(t)=\sum_{i=1}^{n} \lambda_{i}\left[\left(w_{i}^{2}(t)\right)^{\Delta} e_{p}(\sigma(t), 0)+w_{i}^{2}(t) e_{p}^{\Delta}(t, 0)\right] \\
& =\sum_{i=1}^{n} \lambda_{i}\left\{\left[w_{i}(t)+w_{i}(\sigma(t))\right] w_{i}^{\Delta}(t) e_{p}(\sigma(t), 0)\right. \\
& \left.+w_{i}^{2}(t) p e_{p}(t, 0)\right\} \\
& =\sum_{i=1}^{n} \lambda_{i}\left\{\left[2 w_{i}(t) w_{i}^{\Delta}(t)+\mu(t)\left(w_{i}^{\Delta}(t)\right)^{2}\right]\right. \\
& \left.\times e_{p}(\sigma(t), 0)+w_{i}^{2}(t) p e_{p}(t, 0)\right\}
\end{aligned}
$$

$$
\begin{aligned}
\leq \sum_{i=1}^{n} \lambda_{i}\left\{2 w_{i}(t)[\right. & -a_{i} w_{i}(t)+\sum_{j=1}^{n}\left|a_{i j}\right| L_{j}\left|w_{j}(t)\right| \\
& \left.+\sum_{j=1}^{n} \alpha_{i j} L_{j}\left|w_{j}\left(t-\tau_{j}\right)\right|+\left|B_{i}\right|\left|\rho_{i}(t)\right|\right]
\end{aligned}
$$$$
\times e_{p}(\sigma(t), 0)
$$$$
+\mu(t)\left[-a_{i} w_{i}(t)+\sum_{j=1}^{n}\left|a_{i j}\right| L_{j}\left|w_{j}(t)\right|\right.
$$$$
+\sum_{j=1}^{n} \alpha_{i j} L_{j} \times\left|w_{j}\left(t-\tau_{j}\right)\right|
$$$$
\left.+\left|B_{i}\right|\left|\rho_{i}(t)\right|\right]^{2} e_{p}(\sigma(t), 0)
$$$$
\left.+w_{i}^{2}(t) p e_{p}(t, 0)\right\}
$$$$
\leq \sum_{i=1}^{n} \lambda_{i} e_{p}(\sigma(t), 0)
$$$$
\times\left\{\left[-2 a_{i} w_{i}^{2}(t)+\sum_{j=1}^{n}\left|a_{i j}\right| L_{j}\left(w_{i}^{2}(t)+w_{j}^{2}(t)\right)\right.\right.
$$$$
+\sum_{j=1}^{n} \alpha_{i j} L_{j} \times\left(w_{i}^{2}(t)+w_{j}^{2}\left(t-\tau_{j}\right)\right)
$$$$
\left.+\left|B_{i}\right|\left(w_{i}^{2}(t)+\rho_{i}^{2}(t)\right)\right]
$$$$
+\mu(t)\left[a_{i}^{2} w_{i}^{2}(t)+n \sum_{j=1}^{n} a_{i j}^{2} L_{j}^{2} w_{j}^{2}(t)\right.
$$$$
+n \sum_{j=1}^{n} \alpha_{i j}^{2} L_{j}^{2} w_{j}^{2}\left(t-\tau_{j}\right)+B_{i}^{2} \rho_{i}^{2}(t)
$$$$
+a_{i} \sum_{j=1}^{n}\left|a_{i j}\right| L_{j}\left(w_{i}^{2}(t)+w_{j}^{2}(t)\right)
$$$$
+a_{i} \sum_{j=1}^{n} \alpha_{i j} L_{j}\left(w_{i}^{2}(t)+w_{j}^{2}\left(t-\tau_{j}\right)\right)
$$$$
+a_{i}\left|B_{i}\right|\left(w_{i}^{2}(t)+\rho_{i}^{2}(t)\right)
$$ 


$$
\begin{aligned}
& +\sum_{j=1}^{n}\left|a_{i j}\right| \alpha_{i j} L_{j}^{2} \\
& \times\left(w_{j}^{2}(t)+w_{j}^{2}\left(t-\tau_{j}\right)\right) \\
& +\sum_{j=1}^{n}\left|a_{i j}\right| L_{j}\left|B_{i}\right|\left(w_{j}^{2}(t)+\rho_{i}^{2}(t)\right) \\
& +\left|B_{i}\right| \\
& \left.\left.\times \sum_{j=1}^{n} \alpha_{i j} L_{j}\left(w_{j}^{2}\left(t-\tau_{j}\right)+\rho_{i}^{2}(t)\right)\right]\right\} \\
& +\left|B_{i}\right|[1+\mu(t) \\
& \times\left(\left|B_{i}\right|+a_{i}\right. \\
& \left.\left.+\sum_{j=1}^{n}\left(\left|a_{i j}\right|+\alpha_{i j}\right) L_{j}\right)\right] \\
& \left.\times \rho_{i}^{2}(t)\right\} \\
& +\sum_{i=1}^{n} \lambda_{i} p e_{p}(t, 0) w_{i}^{2}(t) \\
& =\sum_{i=1}^{n} \lambda_{i} e_{p}(t, 0) \\
& \times\{p+(1+\mu(t) p) \\
& \times\left[-2 a_{i}+\mu(t) a_{i}^{2}\right. \\
& +\left(\sum_{j=1}^{n}\left(\left|a_{i j}\right|+\alpha_{i j}\right) L_{j}+\left|B_{i}\right|\right) \\
& \left.\left.\times\left(1+\mu(t) a_{i}\right)\right]\right\} w_{i}^{2}(t) \\
& +\sum_{i=1}^{n} \lambda_{i} e_{p}(t, 0)(1+\mu(t) p) \\
& \times\left\{\sum_{j=1}^{n}\left|a_{i j}\right| L_{j}\right. \\
& \times[1+\mu(t) \\
& \left.\times\left(n\left|a_{i j}\right| L_{j}+a_{i}+\alpha_{i j} L_{j}+\left|B_{i}\right|\right)\right] \\
& \times w_{j}^{2}(t) \\
& +\sum_{j=1}^{n} \alpha_{i j} L_{j} \\
& \times[1+\mu(t) \\
& F_{2}^{\Delta}(t)=\sum_{i=1}^{n} \xi_{i}\left[\left(\rho_{i}^{2}(t)\right)^{\Delta} e_{p}(\sigma(t), 0)+\rho_{i}^{2}(t) e_{p}^{\Delta}(t, 0)\right] \\
& =\sum_{i=1}^{n} \xi_{i}\left\{\left[2 \rho_{i}(t) \rho_{i}^{\Delta}(t)+\mu(t)\left(\rho_{i}^{\Delta}(t)\right)^{2}\right]\right. \\
& \left.\times e_{p}(\sigma(t), 0)+\rho_{i}^{2}(t) p e_{p}(t, 0)\right\} \\
& \leq \sum_{i=1}^{n} \xi_{i}\left\{2 \rho _ { i } ( t ) \left[-\alpha_{i} \rho_{i}(t)+\left|\beta_{i}\right| L_{i}\left|w_{i}(t)\right|\right.\right. \\
& \left.+\sum_{j=1}^{n} \beta_{i j} L_{j}\left|w_{j}\left(t-\sigma_{j}\right)\right|\right] \\
& \times e_{p}(\sigma(t), 0) \\
& +\mu(t)\left[-\alpha_{i} \rho_{i}(t)+\left|\beta_{i}\right| L_{i}\left|w_{i}(t)\right|\right. \\
& \left.+\sum_{j=1}^{n} \beta_{i j} L_{j}\left|w_{j}\left(t-\sigma_{j}\right)\right|\right]^{2} \\
& \left.\times e_{p}(\sigma(t), 0)+\rho_{i}^{2}(t) p e_{p}(t, 0)\right\} \\
& \leq \sum_{i=1}^{n} \xi_{i} e_{p}(\sigma(t), 0) \\
& \times\left\{\left[-2 \alpha_{i} \rho_{i}^{2}(t)+\left|\beta_{i}\right| L_{i}\left(\rho_{i}^{2}(t)+w_{i}^{2}(t)\right)\right.\right. \\
& \left.\times\left(n \alpha_{i j} L_{j}+a_{i}+\left|a_{i j}\right| L_{j}+\left|B_{i}\right|\right)\right] \\
& \times w_{j}^{2}\left(t-\tau_{j}\right) \\
& \left.+\sum_{j=1}^{n} \beta_{i j} L_{j}\left(\rho_{i}^{2}(t)+w_{j}^{2}\left(t-\sigma_{j}\right)\right)\right]
\end{aligned}
$$




$$
\begin{aligned}
& +\mu(t)\left[\alpha_{i}^{2} \rho_{i}^{2}(t)+\beta_{i}^{2} L_{i}^{2} w_{i}^{2}(t)\right. \\
& F_{3}^{\Delta}(t)=\sum_{i=1}^{n} \sum_{j=1}^{n} \lambda_{i} e_{p}\left(t+\tau_{j}, 0\right)\left(1+\mu\left(t+\tau_{j}\right) p\right) \\
& \times\left\{\alpha_{i j} L_{j}\right. \\
& +n \sum_{j=1}^{n} \beta_{i j}^{2} L_{j}^{2} w_{j}^{2}\left(t-\sigma_{j}\right) \\
& \times\left[1+\mu\left(t+\tau_{j}\right)\right. \\
& \left.\left.\times\left(n \alpha_{i j} L_{j}+a_{i}+\left|a_{i j}\right| L_{j}+\left|B_{i}\right|\right)\right]\right\} \\
& +\alpha_{i}\left|\beta_{i}\right| L_{i}\left(\rho_{i}^{2}(t)+w_{i}^{2}(t)\right) \\
& +\alpha_{i} L_{j} \sum_{j=1}^{n} \beta_{i j}\left(\rho_{i}^{2}(t)+w_{j}^{2}\left(t-\sigma_{j}\right)\right) \\
& \times w_{j}^{2}(t) \\
& -\sum_{i=1}^{n} \sum_{j=1}^{n} \lambda_{i}(1+\mu(t) p) e_{p}(t, 0) \\
& +\left|\beta_{i}\right| L_{i} \\
& \left.\left.\times \sum_{j=1}^{n} \beta_{i j} L_{j}\left(w_{i}^{2}(t)+w_{j}^{2}\left(t-\sigma_{j}\right)\right)\right]\right\} \\
& +\sum_{i=1}^{n} \xi_{i} p e_{p}(t, 0) \rho_{i}^{2}(t) \\
& =\sum_{i=1}^{n} \xi_{i} e_{p}(t, 0) \\
& \times\{p+(1+\mu(t) p) \\
& \times\left[-2 \alpha_{i}+\mu(t) \alpha_{i}^{2}+\left(\left|\beta_{i}\right| L_{i}+\sum_{j=1}^{n} \beta_{i j} L_{j}\right)\right. \\
& \left.\left.\times\left(1+\mu(t) \alpha_{i}\right)\right]\right\} \rho_{i}^{2}(t) \\
& +\sum_{i=1}^{n} \xi_{i} e_{p}(t, 0)(1+\mu(t) p) \\
& \times\left\{\left|\beta_{i}\right| L_{i}\right. \\
& \times\left\{\alpha_{i j} L_{j}\right. \\
& \times[1+\mu(t) \\
& \left.\left.\times\left(n \alpha_{i j} L_{j}+a_{i}+\left|a_{i j}\right| L_{j}+\left|B_{i}\right|\right)\right]\right\} \\
& \times w_{j}^{2}\left(t-\tau_{j}\right) \\
& F_{4}^{\Delta}(t)=\sum_{i=1}^{n} \sum_{j=1}^{n} \xi_{i} e_{p}\left(t+\sigma_{j}, 0\right)\left(1+\mu\left(t+\sigma_{j}\right) p\right) \\
& \times\left\{\beta _ { i j } L _ { j } \left[1+\mu\left(t+\sigma_{j}\right)\right.\right. \\
& \left.\left.\times\left(n \beta_{i j} L_{j}+\alpha_{i}+\left|\beta_{i}\right| L_{i}\right)\right]\right\} w_{j}^{2}(t) \\
& -\sum_{i=1}^{n} \sum_{j=1}^{n} \xi_{i} e_{p}(t, 0)(1+\mu(t) p) \\
& \times\left\{\beta_{i j} L_{j}[1+\mu(t)\right. \\
& \left.\left.\times\left(n \beta_{i j} L_{j}+\alpha_{i}+\left|\beta_{i}\right| L_{i}\right)\right]\right\} \\
& \times w_{j}^{2}\left(t-\sigma_{j}\right) \\
& F^{\Delta}(t)=F_{1}^{\Delta}(t)+F_{2}^{\Delta}(t)+F_{3}^{\Delta}(t)+F_{4}^{\Delta}(t) \\
& \leq \sum_{i=1}^{n} \lambda_{i} e_{p}(t, 0) \\
& \times\left[1+\mu(t)\left(\left|\beta_{i}\right| L_{i}+\alpha_{i}+\sum_{j=1}^{n} L_{j} \beta_{i j}\right)\right] \\
& \times w_{i}^{2}(t) \\
& +\sum_{j=1}^{n} \beta_{i j} L_{j} \\
& \times\left[1+\mu(t)\left(n \beta_{i j} L_{j}+\alpha_{i}+\left|\beta_{i}\right| L_{i}\right)\right] \\
& \times\{p+(1+\mu(t) p) \\
& \times\left[-2 a_{i}+\mu(t) a_{i}^{2}\right. \\
& +\left(\sum_{j=1}^{n}\left(\left|a_{i j}\right|+\alpha_{i j}\right) L_{j}+\left|B_{i}\right|\right) \\
& \left.\times w_{j}^{2}\left(t-\sigma_{j}\right)\right\} \\
& \left.\left.\times\left(1+\mu(t) a_{i}\right)\right]\right\} w_{i}^{2}(t)
\end{aligned}
$$




$$
\begin{aligned}
& +\sum_{i=1}^{n} \lambda_{i} e_{p}(t, 0)(1+\mu(t) p) \\
& \times\left\{\sum_{j=1}^{n}\left|a_{i j}\right| L_{j}\right. \\
& \times[1+\mu(t) \\
& \left.\times\left(n\left|a_{i j}\right| L_{j}+a_{i}+\alpha_{i j} L_{j}+\left|B_{i}\right|\right)\right] \\
& \times w_{j}^{2}(t) \\
& +\left|B_{i}\right|[1+\mu(t) \\
& \times\left(\left|B_{i}\right|+a_{i}\right. \\
& \left.\left.\left.\times \rho_{i}^{2}(t)\right\}+\sum_{j=1}^{n}\left(\left|a_{i j}\right|+\alpha_{i j}\right) L_{j}\right)\right] \\
& \\
& \quad
\end{aligned}
$$$$
+\sum_{i=1}^{n} \sum_{j=1}^{n} \lambda_{i} e_{p}\left(t+\tau_{j}, 0\right)\left(1+\mu\left(t+\tau_{j}\right) p\right)
$$$$
+\sum_{i=1}^{n} \xi_{i} e_{p}(t, 0)
$$$$
\times\{p+(1+\mu(t) p)
$$$$
\times\left[-2 \alpha_{i}+\mu(t) \alpha_{i}^{2}+\left(\left|\beta_{i}\right| L_{i}+\sum_{j=1}^{n} \beta_{i j} L_{j}\right)\right.
$$$$
\left.\left.\times\left(1+\mu(t) \alpha_{i}\right)\right]\right\} \rho_{i}^{2}(t)
$$

$+\sum_{i=1}^{n} \xi_{i} e_{p}(t, 0)(1+\mu(t) p)$

$$
\begin{aligned}
\times\left\{\left|\beta_{i}\right| L_{i}[1\right. & +\mu(t) \\
& \left.\times\left(\left|\beta_{i}\right| L_{i}+\alpha_{i}+\sum_{j=1}^{n} \beta_{i j} L_{j}\right)\right]
\end{aligned}
$$

$\times w_{i}^{2}(t)$

$$
\begin{aligned}
& \times\left\{\alpha _ { i j } L _ { j } \left[1+\mu\left(t+\tau_{j}\right)\right.\right. \\
& \left.\left.\times\left(n \alpha_{i j} L_{j}+a_{i}+\left|a_{i j}\right| L_{j}+\left|B_{i}\right|\right)\right]\right\} \\
& \times w_{j}^{2}(t) \\
& +\sum_{i=1}^{n} \sum_{j=1}^{n} \xi_{i} e_{p}\left(t+\sigma_{j}, 0\right)\left(1+\mu\left(t+\sigma_{j}\right) p\right) \\
& \times\left\{\beta_{i j} L_{j}\right. \\
& \left.\times\left[1+\mu\left(t+\sigma_{j}\right)\left(n \beta_{i j} L_{j}+\alpha_{i}+\left|\beta_{i}\right| L_{i}\right)\right]\right\} \\
& \times w_{j}^{2}(t) \\
& =\sum_{i=1}^{n} e_{p}(t, 0) \\
& \times\left\{\lambda_{i}[p+(1+\mu(t) p)\right. \\
& \times\left(-2 a_{i}+\mu(t) a_{i}^{2}\right. \\
& +\left(\sum_{j=1}^{n}\left(\left|a_{i j}\right|+\alpha_{i j}\right) L_{j}+\left|B_{i}\right|\right) \\
& \left.\left.\times\left(1+\mu(t) a_{i}\right)\right)\right] \\
& +\xi_{i}(1+\mu(t) p)\left|\beta_{i}\right| L_{i} \\
& \left.\times\left[1+\mu(t)\left(\left|\beta_{i}\right| L_{i}+\alpha_{i}+\sum_{j=1}^{n} \beta_{i j} L_{j}\right)\right]\right\} \\
& \times w_{i}^{2}(t) \\
& +\sum_{i=1}^{n} \sum_{j=1}^{n} e_{p}(t, 0) \\
& \times\left\{\lambda_{i}(1+\mu(t) p)\left|a_{i j}\right| L_{j}\right. \\
& \times[1+\mu(t) \\
& \left.\times\left(n\left|a_{i j}\right| L_{j}+a_{i}+\alpha_{i j} L_{j}+\left|B_{i}\right|\right)\right] \\
& +\lambda_{i}\left(1+\mu\left(t+\tau_{j}\right) p\right) e_{p}\left(t+\tau_{j}, t\right) \alpha_{i j} L_{j}
\end{aligned}
$$




$$
\begin{aligned}
& \times\left[1+\mu\left(t+\tau_{j}\right)\right. \\
& \left.\times\left(n \alpha_{i j} L_{j}+a_{i}+\left|a_{i j}\right| L_{j}+\left|B_{i}\right|\right)\right] \\
& +\xi_{i}\left(1+\mu\left(t+\sigma_{j}\right) p\right) \\
& \times e_{p}\left(t+\sigma_{j}, t\right) \beta_{i j} L_{j} \\
& \times\left[1+\mu\left(t+\sigma_{j}\right)\right. \\
& \left.\left.\times\left(n \beta_{i j} L_{j}+\alpha_{i}+\left|\beta_{i}\right| L_{i}\right)\right]\right\} \\
& \times w_{j}^{2}(t) \\
& +\sum_{i=1}^{n} e_{p}(t, 0) \\
& \times\left\{\lambda_{i}(1+\mu(t) p)\left|B_{i}\right|\right. \\
& \times[1+\mu(t) \\
& \left.\times\left(\left|B_{i}\right|+a_{i}+\sum_{j=1}^{n}\left(\left|a_{i j}\right|+\alpha_{i j}\right) L_{j}\right)\right] \\
& +\xi_{i}[p+(1+\mu(t) p) \\
& \times\left(-2 \alpha_{i}+\mu(t) \alpha_{i}^{2}\right. \\
& +\left(\left|\beta_{i}\right| L_{i}+\sum_{j=1}^{n} \beta_{i j} L_{j}\right) \\
& \left.\left.\left.\times\left(1+\mu(t) \alpha_{i}\right)\right)\right]\right\} \rho_{i}^{2}(t) \\
& =\sum_{i=1}^{n} e_{p}(t, 0) \\
& \times\left\{\lambda_{i}[p+(1+\mu(t) p)\right. \\
& \times\left(-2 a_{i}+\mu(t) a_{i}^{2}\right. \\
& +\left(\sum_{j=1}^{n}\left(\left|a_{i j}\right|+\alpha_{i j}\right) L_{j}+\left|B_{i}\right|\right) \\
& \left.\left.\times\left(1+\mu(t) a_{i}\right)\right)\right] \\
& +\xi_{i}(1+\mu(t) p)\left|\beta_{i}\right| L_{i} \\
& \times\left[1+\mu(t)\left(\left|\beta_{i}\right| L_{i}+\alpha_{i}+\sum_{j=1}^{n} \beta_{i j} L_{j}\right)\right] \\
& +\sum_{j=1}^{n} \lambda_{j}(1+\mu(t) p)\left|a_{j i}\right| L_{i} \\
& \times[1+\mu(t) \\
& \left.\times\left(n\left|a_{j i}\right| L_{i}+a_{j}+\alpha_{j i} L_{i}+\left|B_{j}\right|\right)\right] \\
& +\sum_{j=1}^{n} \lambda_{j} \times\left(1+\mu\left(t+\tau_{i}\right) p\right) \\
& \times e_{p}\left(t+\tau_{i}, t\right) \alpha_{j i} L_{i} \\
& \times\left[1+\mu\left(t+\tau_{i}\right)\right. \\
& \left.\times\left(n \alpha_{j i} L_{i}+a_{j}+\left|a_{j i}\right| L_{i}+\left|B_{j}\right|\right)\right] \\
& +\sum_{j=1}^{n} \xi_{j}\left(1+\mu\left(t+\sigma_{i}\right) p\right) e_{p}\left(t+\sigma_{i}, t\right) \beta_{j i} L_{i} \\
& \times\left[1+\mu\left(t+\sigma_{i}\right)\right. \\
& \left.\left.\times\left(n \beta_{j i} L_{i}+\alpha_{j}+\left|\beta_{j}\right| L_{j}\right)\right]\right\} w_{i}^{2}(t) \\
& +\sum_{i=1}^{n} e_{p}(t, 0) \\
& \times\left\{\lambda_{i}(1+\mu(t) p)\left|B_{i}\right|\right. \\
& \times[1+\mu(t) \\
& \left.\times\left(\left|B_{i}\right|+a_{i}+\sum_{j=1}^{n}\left|a_{i j}\right| L_{j}+\sum_{j=1}^{n} \alpha_{i j} L_{j}\right)\right] \\
& +\xi_{i}[p+(1+\mu(t) p) \\
& \times\left(-2 \alpha_{i}+\mu(t) \alpha_{i}^{2}\right. \\
& +\left(\left|\beta_{i}\right| L_{i}+\sum_{j=1}^{n} \beta_{i j} L_{j}\right) \\
& \left.\left.\left.\times\left(1+\mu(t) \alpha_{i}\right)\right)\right]\right\} \rho_{i}^{2}(t)
\end{aligned}
$$




$$
\begin{aligned}
& =\sum_{i=1}^{n} e_{p}(t, 0) \\
& +\sum_{i=1}^{n} e_{p}(t, 0) \\
& \times\left\{\lambda_{i} p+(1+\mu(t) p)\right. \\
& \times\left\{\xi_{i} p+(1+\mu(t) p)\right. \\
& \times\left[-2 \lambda_{i} a_{i}\right. \\
& +\lambda_{i}\left(\sum_{j=1}^{n}\left(\left|a_{i j}\right|+\alpha_{i j}\right) L_{j}+\left|B_{i}\right|\right) \\
& +\xi_{i}\left|\beta_{i}\right| L_{i}+\sum_{j=1}^{n} \lambda_{j}\left|a_{j i}\right| L_{i} \\
& +\mu(t)\left[\lambda_{i} a_{i}^{2}\right. \\
& +\lambda_{i} a_{i} \\
& \times\left(\sum_{j=1}^{n}\left(\left|a_{i j}\right|+\alpha_{i j}\right) L_{j}+\left|B_{i}\right|\right) \\
& +\xi_{i}\left|\beta_{i}\right| L_{i} \\
& \times\left(\left|\beta_{i}\right| L_{i}+\alpha_{i}+\sum_{j=1}^{n} \beta_{i j} L_{j}\right) \\
& +\sum_{j=1}^{n} \lambda_{j}\left|a_{j i}\right| L_{i} \\
& \times\left(n\left|a_{j i}\right| L_{i}+a_{j}+\alpha_{j i} L_{i}\right. \\
& \left.\left.\left.+\left|B_{j}\right|\right)\right]\right] \\
& +\sum_{j=1}^{n} \lambda_{j}\left(1+\mu\left(t+\tau_{i}\right) p\right) \\
& \times e_{p}\left(t+\tau_{i}, t\right) \alpha_{j i} L_{i} \\
& \times\left[1+\mu\left(t+\tau_{i}\right)\right. \\
& \left.\times\left(n \alpha_{j i} L_{i}+a_{j}+\left|a_{j i}\right| L_{i}+\left|B_{j}\right|\right)\right] \\
& +\sum_{j=1}^{n} \xi_{j}\left(1+\mu\left(t+\sigma_{i}\right) p\right) \\
& \times e_{p}\left(t+\sigma_{i}, t\right) \beta_{j i} L_{i} \\
& \times\left[1+\mu\left(t+\sigma_{i}\right)\right. \\
& \left.\left.\times z\left(n \beta_{j i} L_{i}+\alpha_{j}+\left|\beta_{j}\right| L_{j}\right)\right]\right\} w_{i}^{2}(t) \\
& \times\left[\lambda_{i}\left|B_{i}\right|-2 \xi_{i} \alpha_{i}+\xi_{i}\left|\beta_{i}\right| L_{i}\right. \\
& +\xi_{i} \sum_{j=1}^{n} \beta_{i j} L_{j}+\mu(t) \\
& \times\left[\lambda _ { i } | B _ { i } | \left(\left|B_{i}\right|+a_{i}\right.\right. \\
& \left.+\sum_{j=1}^{n}\left(\left|a_{i j}\right|+\alpha_{i j}\right) L_{j}\right) \\
& +\alpha_{i} \xi_{i}\left(\alpha_{i}+\left|\beta_{i}\right| L_{i}\right. \\
& \left.\left.\left.\left.+\sum_{j=1}^{n} \beta_{i j} L_{j}\right)\right]\right]\right\} \rho_{i}^{2}(t) . \\
& F(0)=F_{1}(0)+F_{2}(0)+F_{3}(0)+F_{4}(0) \\
& \leq \sum_{i=1}^{n}\left\{\lambda_{i}\right. \\
& +\sum_{j=1}^{n} \lambda_{j} \alpha_{j i} L_{i} \\
& \times\left[1+\mu\left(n \alpha_{j i} L_{i}+a_{j}+\left|a_{j i}\right| L_{i}+\left|B_{j}\right|\right)\right] \\
& \times\left(1+p \mu^{*}\right) \int_{-\tau_{i}}^{0} e_{p}\left(s+\tau_{i}, 0\right) \Delta s \\
& +\sum_{j=1}^{n} \xi_{j} \beta_{j i} L_{i} \\
& \times\left[1+\mu\left(n \beta_{j i} L_{i}+\alpha_{j}+\left|\beta_{j}\right| L_{j}\right)\right] \\
& \left.\times\left(1+p \mu^{*}\right) \int_{-\sigma_{i}}^{0} e_{p}\left(s+\sigma_{i}, 0\right) \Delta s\right\}
\end{aligned}
$$




$$
\begin{aligned}
& \times \sup _{-\theta \leq s \leq 0} w_{i}^{2}(s)+\sum_{i=1}^{n} \xi_{i} \sup _{-\theta \leq l \leq 0} \rho_{i}^{2}(l) \\
& \leq \max _{1 \leq i \leq n}\left\{\lambda_{i}\right. \\
& +\sum_{j=1}^{n} \lambda_{j} \alpha_{j i} L_{i} \\
& \times\left[1+\mu\left(n \alpha_{j i} L_{i}+a_{j}+\left|a_{j i}\right| L_{i}\left|B_{j}\right|\right)\right] \\
& \times(1+p \mu) \tau e_{p}(\tau, 0) \\
& +\sum_{j=1}^{n} \xi_{j} \beta_{j i} L_{i}\left[1+\mu\left(n \beta_{j i} L_{i}+\alpha_{j}\left|\beta_{j}\right| L_{j}\right)\right] \\
& \left.\times(1+p \mu) \sigma e_{p}(\sigma, 0)\right\} \sum_{i=1}^{n} \sup _{-\theta \leq s \leq 0} w_{i}^{2}(s) \\
& +\max _{1 \leq i \leq n} \xi_{i} \sum_{i=1}^{n} \sup _{-\theta \leq l \leq 0} \rho_{i}^{2}(l) \leq M_{1} \Phi_{\theta},
\end{aligned}
$$

where $|\mu(t)| \leq \mu^{*}, \tau=\max _{1 \leq j \leq n}\left(\tau_{j}\right), \sigma=\max _{1 \leq j \leq n}\left(\sigma_{j}\right), \theta=$ $\max _{1 \leq j \leq n}\left(\tau_{j}, \sigma_{j}\right)$, and

$$
\begin{aligned}
M_{1}=\max _{1 \leq i \leq n}\left\{\operatorname { m a x } _ { 1 \leq i \leq n } \left\{\begin{array}{l}
\lambda_{i} \\
+
\end{array}\right.\right. & \sum_{j=1}^{n} \lambda_{j} \alpha_{j i} L_{i} \\
& \times\left[1+\mu\left(n \alpha_{j i} L_{i}+a_{j}+\left|a_{j i}\right| L_{i}\left|B_{j}\right|\right)\right] \\
& \times(1+p \mu) \tau e_{p}(\tau, 0) \\
+ & \sum_{j=1}^{n} \xi_{j} \beta_{j i} L_{i} \\
& \times\left[1+\mu\left(n \beta_{j i} L_{i}+\alpha_{j}\left|\beta_{j}\right| L_{j}\right)\right] \\
& \left.\left.\times(1+p \mu) \sigma e_{p}(\sigma, 0)\right\}, \max _{1 \leq i \leq n}\right\} .
\end{aligned}
$$

Observe that

$$
\begin{aligned}
F(t) & \geq \min _{1 \leq i \leq n} \lambda_{i} \sum_{i=1}^{n} w_{i}^{2}(t) e_{p}(t, 0)+\min _{1 \leq i \leq n} \xi_{i} \sum_{i=1}^{n} \rho_{i}^{2}(t) e_{p}(t, 0) \\
& \geq M_{2}\left(\sum_{i=1}^{n} w_{i}^{2}(t)+\sum_{i=1}^{n} \rho_{i}^{2}(t)\right) e_{p}(t, 0)
\end{aligned}
$$

where $M_{2}=\min _{1 \leq j \leq n}\left\{\min _{1 \leq i \leq n} \lambda_{i}, \min _{1 \leq i \leq n} \xi_{i}\right\}$. Due to Lemma 11, we get

$$
\begin{array}{r}
\sum_{i=1}^{n} w_{i}^{2}(t)+\sum_{i=1}^{n} \rho_{i}^{2}(t) \leq M \cdot e_{\ominus p}(t, 0) \Phi_{\theta} \longrightarrow 0 \\
\text { as } t \longrightarrow+\infty
\end{array}
$$

where $M=M_{1} / M_{2}>0$ is a constant. This completes the proof.

Remark 15. It is well known that few works have been done to report learning dynamics of fuzzy networks in the literature. If the time scale $\mathbb{T}=\mathbb{R}$, then $\mu(t)=0$ and (4) reduces to common fuzzy networks with learning behavior reported by [16]. Removing learning variables, (4) is a generation form of $[2,4,7]$. From Theorem 14, we can immediately obtain the following corollary which contains relative results in [16].

Corollary 16. Suppose that (4) satisfies $\left(\mathscr{A}_{1}\right), \mathbb{\mathbb { V }}=\mathbb{R}$, and if there exist constants $\lambda_{i}>0, \xi_{i}>0$ such that

$$
\begin{gathered}
\lambda_{i}\left[-2 a_{i}+\sum_{j=1}^{n}\left(\left|a_{i j}\right|+\alpha_{i j}\right) L_{j}+\left|B_{i}\right|\right]+\xi_{i}\left|\beta_{i}\right| L_{i} \\
\quad+\sum_{j=1}^{n} \lambda_{j}\left|a_{j i}\right| L_{i}+\sum_{j=1}^{n} \lambda_{j} \alpha_{j i} L_{i}+\sum_{j=1}^{n} \xi_{j} \beta_{j i} L_{i}<0, \\
\lambda_{i}\left|B_{i}\right|+\xi_{i}\left[-2 \alpha_{i}+\left|\beta_{i}\right| L_{i}+\sum_{j=1}^{n} \beta_{i j} L_{j}\right]<0,
\end{gathered}
$$

where $i \in \mathbb{N}$, then there exists a unique equilibrium $\left(u^{*}, v^{*}\right)$ of (4) which is globally exponentially stable.

If the time scale $\mathbb{T}=Z$, then $\mu(t)=1$ and (4) reduces to

$$
\begin{aligned}
u_{i}(n+1)= & \left(1-a_{i}\right) u_{i}(n)+\sum_{j=1}^{n} a_{i j} f_{j}\left(u_{j}(n)\right) \\
& +\bigvee_{j=1}^{n} b_{i j}^{\ell} f_{j}\left(u_{j}\left(n-\tau_{j}\right)\right) \\
& +\bigwedge_{j=1}^{n} c_{i j}^{\ell} f_{j}\left(u_{j}\left(n-\tau_{j}\right)\right)+B_{i} v_{i}(n)+J_{i}, \\
v_{i}(n+1)= & \left(1-\alpha_{i}\right) v_{i}(n)+\beta_{i} f_{i}\left(u_{i}(t)\right) \\
& +\bigwedge_{j=1}^{n} \gamma_{i j}^{\ell} f_{j}\left(u_{j}\left(n-\sigma_{j}\right)\right) \\
& +\bigvee_{j=1}^{n} \delta_{i j}^{\ell} f_{j}\left(u_{j}\left(n-\sigma_{j}\right)\right),
\end{aligned}
$$

which includes discrete-time analogues of competitive networks [24] as its special case when there is no fuzzy terms. From Theorem 14, we have the following corollary. 
Corollary 17. Suppose that (25) satisfies $\left(\mathscr{A}_{1}\right)$ and if there exist constants $\lambda_{i}>0, \xi_{i}>0$ such that

$$
\begin{aligned}
\lambda_{i}[ & \left.-2 a_{i}+a_{i}^{2}+\left(\sum_{j=1}^{n}\left(\left|a_{i j}\right|+\alpha_{i j}\right) L_{j}+\left|B_{i}\right|\right)\left(1+a_{i}\right)\right] \\
& +\xi_{i}\left|\beta_{i}\right| L_{i}\left[1+\left|\beta_{i}\right| L_{i}+\alpha_{i}+\sum_{j=1}^{n} \beta_{i j} L_{j}\right] \\
& +\sum_{j=1}^{n} \lambda_{j}\left|a_{j i}\right| L_{i}\left[1+n\left|a_{j i}\right| L_{i}+a_{j}+\alpha_{j i} L_{i}+\left|B_{j}\right|\right] \\
+ & \sum_{j=1}^{n} \lambda_{j} \alpha_{j i} L_{i} \\
& \times\left[1+n \alpha_{j i} L_{i}+a_{j}+\left|a_{j i}\right| L_{i}+\left|B_{j}\right|\right] \\
+ & \sum_{j=1}^{n} \xi_{j} \beta_{j i} L_{i}\left[1+n \beta_{j i} L_{i}+\alpha_{j}+\left|\beta_{j}\right| L_{j}\right]<0, \\
& <\xi_{i}\left[-2 \alpha_{i}+\alpha_{i}^{2}+\left(\left|\beta_{i}\right| L_{i}+\sum_{j=1}^{n} \beta_{i j} L_{j}\right)\left(1+\alpha_{i}\right)\right] \\
\lambda_{i}\left|B_{i}\right| & {\left[1+\left|B_{i}\right|+a_{i}+\sum_{j=1}^{n}\left|a_{i j}\right| L_{j}+\sum_{j=1}^{n} \alpha_{i j} L_{j}\right] }
\end{aligned}
$$

where $i \in \mathbb{N}$, then there exists a unique equilibrium $\left(u^{*}, v^{*}\right)$ of (25) which is globally exponentially stable.

Remark 18. The result of Theorem 14 unifies the previous literature on fuzzy networks of discrete-time and continuoustime and reveals the discrepancies of results of continuoustime $(\mu(t)=0)$ and discrete-time $(\mu(t)=1)$ fuzzy networks $[1-4,16]$.

Finally, we should briefly investigate learning convergence of fuzzy networks. Consider learning dynamics governed by (5):

$$
\begin{aligned}
m_{i j}^{\Delta}(t)= & -\alpha_{i} m_{i j}(t) \\
+ & {\left[\beta_{i} f_{i}\left(u_{i}(t)\right)+\bigwedge_{k=1}^{n} \gamma_{i k}^{\ell} f_{k}\left(u_{k}\left(t-\sigma_{k}\right)\right)\right.} \\
& \left.+\bigvee_{k=1}^{n} \delta_{i k}^{\ell} f_{k}\left(u_{k}\left(t-\sigma_{k}\right)\right)\right] p_{j},
\end{aligned}
$$

where $i, j \in \mathbb{N}$ and $t \in \mathbb{T}_{0}^{+}$. It follows from Theorem 14 that (4) has a unique equilibrium $\left(u^{*}, v^{*}\right)=\left(u_{1}^{*}, \ldots, u_{n}^{*}, v_{1}^{*}, \ldots, v_{n}^{*}\right)$. Hence, (5) has a unique equilibrium satisfying

$$
m_{i j}^{*}=\frac{p_{j}}{\alpha_{i}}\left[\beta_{i} f_{i}\left(u_{i}^{*}\right)+\bigwedge_{k=1}^{n} \gamma_{i k}^{\ell} f_{k}\left(u_{k}^{*}\right)+\bigvee_{k=1}^{n} \delta_{i k}^{\ell} f_{k}\left(u_{k}^{*}\right)\right] \text {, }
$$

By (23), there exists a $\widetilde{M}>0$ such that

$$
\left|u_{i}(t)-u_{i}^{*}\right| \leq \widetilde{M} \sqrt{e_{\ominus p}(t, 0)}, \quad i \in \mathbb{N} \text {. }
$$

It follows from (5), (28), and (29) that we get

$$
\begin{aligned}
\left(m_{i j}(t)-m_{i j}^{*}\right)^{\Delta} \leq & -\alpha_{i}\left(m_{i j}(t)-m_{i j}^{*}\right) \\
& +\left|p_{j}\right|\left|\beta_{i}\right| L_{i}\left|u_{i}(t)-u_{i}^{*}\right| \\
& +\left|p_{j}\right| \sum_{k=1}^{n} \beta_{i k} L_{k}\left|u_{k}\left(t-\sigma_{k}\right)-u_{k}^{*}\right| \\
\leq & -\alpha_{i}\left(m_{i j}(t)-m_{i j}^{*}\right) \\
& +\widetilde{M}\left[\left|p_{j}\right|\left|\beta_{i}\right| L_{i} \sqrt{e_{\ominus p}\left(t, t-\sigma_{k}\right)}\right. \\
& \left.+\sum_{k=1}^{n}\left|p_{j}\right| \beta_{i k} L_{k}\right] \\
& \times \sqrt{e_{\ominus p}\left(t-\sigma_{k}, 0\right) .}
\end{aligned}
$$

Let $\mu^{M}:=\sup _{s \in \mathbb{T}} \mu(s)$. Since $F(\mu)=-\log (1+p \mu) / \mu$ is an increasing function defined on $\mathbb{R}^{+}$, we know $\xi_{\mu(\tau)}(\ominus p) \leq$ $\max \left\{\ominus p,-\log \left(1+p \mu^{M}\right) / \mu^{M}\right\}: \triangleq \widetilde{N}$ and

$$
\begin{gathered}
\sqrt{e_{\ominus p}\left(t, t-\sigma_{k}\right)} \leq \sqrt{\exp \left(\widetilde{N} \sigma_{k}\right)}, \\
\sqrt{e_{\ominus p}(t, 0)} \leq \sqrt{\exp (\widetilde{N} t)} .
\end{gathered}
$$

Hence, for any given $\epsilon>0$, there exists a $t_{0} \in \mathbb{T}$ such that $\sqrt{e_{\ominus p}(t, 0)} \leq \epsilon$ as $t \geq t_{0}$. From (30) and Lemma 13, one gets

$$
\begin{aligned}
& m_{i j}(t)-m_{i j}^{*} \\
& \leq\left(m_{i j}\left(t_{0}\right)-m_{i j}^{*}\right) e_{-\alpha_{i}}\left(t, t_{0}\right) \\
&+\widetilde{M} \epsilon\left[\left|p_{j}\right|\left|\beta_{i}\right| L_{i} \sqrt{\exp \left(\widetilde{N} \sigma_{k}\right)}+\sum_{k=1}^{n}\left|p_{j}\right| \beta_{i k} L_{k}\right] \\
& \quad \times \int_{t_{0}}^{t} e_{-\alpha_{i}}(t, \sigma(s)) \Delta s .
\end{aligned}
$$


It follows from Lemma 11 that we have

$$
\begin{aligned}
m_{i j}( & t)-m_{i j}^{*} \\
\leq & \left(m_{i j}\left(t_{0}\right)-m_{i j}^{*}\right) e_{-\alpha_{i}}\left(t, t_{0}\right) \\
& -\frac{\widetilde{M} \epsilon}{\alpha_{i}}\left[\left|p_{j}\right|\left|\beta_{i}\right| L_{i} \sqrt{\exp \left(\widetilde{N} \sigma_{k}\right)}+\sum_{k=1}^{n}\left|p_{j}\right| \beta_{i k} L_{k}\right] \\
& \times\left(e_{-\alpha_{i}}\left(t, t_{0}\right)-1\right) \\
\leq & \left(m_{i j}\left(t_{0}\right)-m_{i j}^{*}\right) e^{-\alpha_{i}\left(t-t_{0}\right)} \\
& -\frac{\widetilde{M} \epsilon}{\alpha_{i}}\left[\left|p_{j}\right|\left|\beta_{i}\right| L_{i} \sqrt{\exp \left(\widetilde{N} \sigma_{k}\right)}+\sum_{k=1}^{n}\left|p_{j}\right| \beta_{i k} L_{k}\right] \\
& \times\left(e^{-\alpha_{i}\left(t-t_{0}\right)}-1\right)
\end{aligned}
$$

which leads to $m_{i j}(t)-m_{i j}^{*} \rightarrow 0$ as $t \rightarrow+\infty$. That is, timevarying and learnable synaptic weights converge exponentially to stationary weights $m_{i j}^{*}$ encoding the signal vector $p=$ $\left(p_{1}, p_{2}, \ldots, p_{n}\right)$ in the sense of (28).

\section{An Example}

In this section, an example is shown to verify the effectiveness of the result obtained in the previous section. Consider the following fuzzy networks with delays on time scale $\mathbb{T}$ :

$$
\begin{aligned}
u_{i}^{\Delta}(t)= & -a_{i} u_{i}(t)+\sum_{j=1}^{2} a_{i j} f_{j}\left(u_{j}(t)\right)+\bigvee_{j=1}^{2} b_{i j}^{\ell} f_{j}\left(u_{j}\left(t-\tau_{j}\right)\right) \\
& +\bigwedge_{j=1}^{2} c_{i j}^{\ell} f_{j}\left(u_{j}\left(t-\tau_{j}\right)\right)+B_{i} v_{i}(t)+J_{i}, \\
v_{i}^{\Delta}(t)= & -\alpha_{i} v_{i}(t)+\beta_{i} f_{i}\left(u_{i}(t)\right)+\bigwedge_{j=1}^{2} \gamma_{i j}^{\ell} f_{j}\left(u_{j}\left(t-\sigma_{j}\right)\right) \\
& +\bigvee_{j=1}^{2} \delta_{i j}^{\ell} f_{j}\left(u_{j}\left(t-\sigma_{j}\right)\right)
\end{aligned}
$$

for $i=1,2$ and $t \in \mathbb{T}_{0}^{+}$, where $\left(a_{1}, a_{2}\right)^{T}=(0.3,0.3)^{T}$, $\left(\alpha_{1}, \alpha_{2}\right)^{T}=(0.2,0.2)^{T},\left(B_{1}, B_{2}\right)^{T}=(0.1,0.1)^{T},\left(\beta_{1}, \beta_{2}\right)^{T}=$ $(0.01,0.02)^{T}, f_{i}(x)=(1 / 2)(|x+1|+|x-1|)$, and

$$
\begin{aligned}
& \left(\begin{array}{ll}
a_{11} & a_{12} \\
a_{21} & a_{22}
\end{array}\right)=\left(\begin{array}{ll}
0.01 & 0.01 \\
0.01 & 0.01
\end{array}\right), \\
& \left(\begin{array}{ll}
b_{11}^{\ell} & b_{12}^{\ell} \\
b_{21}^{\ell} & b_{22}^{\ell}
\end{array}\right)=\left(\begin{array}{ll}
0.01 & 0.02 \\
0.02 & 0.01
\end{array}\right), \\
& \left(\begin{array}{ll}
c_{11}^{\ell} & c_{12}^{\ell} \\
c_{21}^{\ell} & c_{22}^{\ell}
\end{array}\right)=\left(\begin{array}{ll}
0.02 & 0.01 \\
0.01 & 0.02
\end{array}\right),
\end{aligned}
$$

$$
\begin{gathered}
\left(\begin{array}{ll}
\gamma_{11}^{\ell} & \gamma_{12}^{\ell} \\
\gamma_{21}^{\ell} & \gamma_{22}^{\ell}
\end{array}\right)=\left(\begin{array}{ll}
0.02 & 0.02 \\
0.02 & 0.02
\end{array}\right), \\
\left(\begin{array}{ll}
\delta_{11}^{\ell} & \delta_{12}^{\ell} \\
\delta_{21}^{\ell} & \delta_{22}^{\ell}
\end{array}\right)=\left(\begin{array}{ll}
0.01 & 0.01 \\
0.02 & 0.02
\end{array}\right), \\
\tau_{i}=\sigma_{i}=\frac{1}{2}, \quad L_{i}=J_{i}=c=1 .
\end{gathered}
$$

Choosing $\lambda_{1} \xi_{i}=1(i=1,2)$, we can easily verify that the assumptions of Corollaries 16 and 17 are all satisfied, respectively. When $\mathbb{T}=\mathbb{R}$, that is, $\mu(t)=0$,

$$
\begin{aligned}
& \lambda_{1}\left[-2 a_{1}+\sum_{j=1}^{2}\left|a_{1 j}\right| L_{j}+\sum_{j=1}^{2}\left(\left|b_{1 j}^{\ell}\right|+\left|c_{1 j}^{\ell}\right|\right) L_{j}+\left|B_{1}\right|\right] \\
& +\xi_{1}\left|\beta_{1}\right| L_{1}+\sum_{j=1}^{2} \lambda_{j}\left|a_{j 1}\right| L_{1}+\sum_{j=1}^{2} \lambda_{j}\left(\left|b_{j 1}^{\ell}\right|+\left|c_{j 1}^{\ell}\right|\right) L_{1} \\
& \quad+\sum_{j=1}^{2} \xi_{j}\left(\left|\gamma_{j 1}^{\ell}\right|+\left|\delta_{j 1}^{\ell}\right|\right) L_{1}=-0.26<0, \\
& \lambda_{2}\left[-2 a_{2}+\sum_{j=1}^{2}\left|a_{2 j}\right| L_{j}+\sum_{j=1}^{2}\left(\left|b_{2 j}^{\ell}\right|+\left|c_{2 j}^{\ell}\right|\right) L_{j}+\left|B_{2}\right|\right] \\
& \quad+\xi_{2}\left|\beta_{2}\right| L_{2}+\sum_{j=1}^{2} \lambda_{j}\left|a_{j 2}\right| L_{2}+\sum_{j=1}^{2} \lambda_{j}\left(\left|b_{j 2}^{\ell}\right|+\left|c_{j 2}^{\ell}\right|\right) L_{2} \\
& \quad+\sum_{j=1}^{2} \xi_{j}\left(\left|\gamma_{j 2}^{\ell}\right|+\left|\delta_{j 2}^{\ell}\right|\right) L_{2}=-0.25<0, \\
& \lambda_{1}\left|B_{1}\right|+\xi_{1}\left[-2 \alpha_{1}+\left|\beta_{1}\right| L_{1}+\sum_{j=1}^{2}\left(\left|\gamma_{1 j}^{\ell}\right|+\left|\delta_{1 j}^{\ell}\right|\right) L_{j}\right] \\
& \quad=-0.23<0, \\
& \lambda_{2}\left|B_{2}\right|+\xi_{2}\left[-2 \alpha_{2}+\left|\beta_{2}\right| L_{2}+\sum_{j=1}^{2}\left(\left|\gamma_{2 j}^{\ell}\right|+\left|\delta_{2 j}^{\ell}\right|\right) L_{j}\right] \\
& \quad=-0.2<0 .
\end{aligned}
$$

When $\mathbb{T}=\mathbb{Z}$, that is, $\mu(t)=1$,

$$
\begin{aligned}
\lambda_{1}[ & -2 a_{1}+a_{1}^{2} \\
& +\left(\sum_{j=1}^{2}\left|a_{1 j}\right| L_{j}+\sum_{j=1}^{2}\left(\left|b_{1 j}^{\ell}\right|+\left|c_{1 j}^{\ell}\right|\right) L_{j}+\left|B_{1}\right|\right) \\
& \left.\times\left(1+a_{1}\right)\right]+\xi_{1}\left|\beta_{1}\right| L_{1}
\end{aligned}
$$




$$
\begin{aligned}
& \times\left[1+\left|\beta_{1}\right| L_{1}+\alpha_{1}+\sum_{j=1}^{2}\left(\left|\gamma_{1 j}^{\ell}\right|+\left|\delta_{1 j}^{\ell}\right|\right) L_{j}\right] \\
& +\sum_{j=1}^{2} \lambda_{j}\left|a_{j 1}\right| L_{1} \\
& \times\left[1+2\left|a_{j 1}\right| L_{1}+a_{j}+\left(\left|b_{j 1}^{\ell}\right|+\left|c_{j 1}^{\ell}\right|\right) L_{1}+\left|B_{j}\right|\right] \\
& +\sum_{j=1}^{2} \lambda_{j}\left(\left|b_{j 1}^{\ell}\right|+\left|c_{j 1}^{\ell}\right|\right) L_{1} \\
& \times\left[1+2\left(\left|b_{j 1}^{\ell}\right|+\left|c_{j 1}^{\ell}\right|\right) L_{1}+a_{j}+\left|a_{j 1}\right| L_{1}+\left|B_{j}\right|\right] \\
& +\sum_{j=1}^{2} \xi_{j}\left(\left|\gamma_{j 1}^{\ell}\right|+\left|\delta_{j 1}^{\ell}\right|\right) \\
& \times L_{1}\left[1+2\left(\left|\gamma_{j 1}^{\ell}\right|+\left|\delta_{j 1}^{\ell}\right|\right) L_{1}+\alpha_{j}+\left|\beta_{j}\right| L_{j}\right] \\
& =-0.056<0, \\
& \lambda_{2}\left[-2 a_{2}+a_{2}^{2}\right. \\
& +\left(\sum_{j=1}^{2}\left|a_{2 j}\right| L_{j}+\sum_{j=1}^{2}\left(\left|b_{2 j}^{\ell}\right|+\left|c_{2 j}^{\ell}\right|\right) L_{j}+\left|B_{2}\right|\right) \\
& \left.\times\left(1+a_{2}\right)\right] \\
& +\xi_{2}\left|\beta_{2}\right| L_{2}\left[1+\left|\beta_{2}\right| L_{2}+\alpha_{2}+\sum_{j=1}^{2}\left(\left|\gamma_{2 j}^{\ell}\right|+\left|\delta_{2 j}^{\ell}\right|\right) L_{j}\right] \\
& +\sum_{j=1}^{2} \lambda_{j}\left|a_{j 2}\right| L_{2} \\
& \times\left[1+2\left|a_{j 2}\right| L_{2}+a_{j}+\left(\left|b_{j 2}^{\ell}\right|+\left|c_{j 2}^{\ell}\right|\right) L_{2}+\left|B_{j}\right|\right] \\
& +\sum_{j=1}^{2} \lambda_{j}\left(\left|b_{j 2}^{\ell}\right|+\left|c_{j 2}^{\ell}\right|\right) L_{2} \\
& \times\left[1+2\left(\left|b_{j 2}^{\ell}\right|+\left|c_{j 2}^{\ell}\right|\right) L_{2}+a_{j}+\left|a_{j 2}\right| L_{2}+\left|B_{j}\right|\right] \\
& +\sum_{j=1}^{2} \xi_{j}\left(\left|\gamma_{j 2}^{\ell}\right|+\left|\delta_{j 2}^{\ell}\right|\right) \\
& \times L_{2}\left[1+2\left(\left|\gamma_{j 2}^{\ell}\right|+\left|\delta_{j 2}^{\ell}\right|\right) L_{2}+\alpha_{j}+\left|\beta_{j}\right| L_{j}\right] \\
& =-0.0427<0 \text {, }
\end{aligned}
$$$$
\lambda_{1}\left|B_{1}\right|\left[1+\left|B_{1}\right|+a_{1}+\sum_{j=1}^{2}\left|a_{1 j}\right| L_{j}+\sum_{j=1}^{2}\left(\left|b_{1 j}^{\ell}\right|+\left|c_{1 j}^{\ell}\right|\right) L_{j}\right]
$$

$$
\begin{aligned}
+\xi_{1} & {\left[-2 \alpha_{1}+\alpha_{1}^{2}\right.} \\
& \left.+\left(\left|\beta_{1}\right| L_{1}+\sum_{j=1}^{2}\left(\left|\gamma_{1 j}^{\ell}\right|+\left|\delta_{1 j}^{\ell}\right|\right) L_{j}\right)\left(1+\alpha_{1}\right)\right] \\
= & -0.128<0, \\
\lambda_{2}\left|B_{2}\right| & {\left[1+\left|B_{2}\right|+a_{2}+\sum_{j=1}^{2}\left|a_{2 j}\right| L_{j}+\sum_{j=1}^{2}\left(\left|b_{2 j}^{\ell}\right|+\left|c_{2 j}^{\ell}\right|\right) L_{j}\right] } \\
+\xi_{2}\left[-2 \alpha_{2}+\alpha_{2}^{2}\right. & \\
& \left.+\left(\left|\beta_{2}\right| L_{2}+\sum_{j=1}^{2}\left(\left|\gamma_{2 j}^{\ell}\right|+\left|\delta_{2 j}^{\ell}\right|\right) L_{j}\right)\left(1+\alpha_{2}\right)\right] \\
= & -0.092<0 .
\end{aligned}
$$

It follows from Corollaries 16 and 17 that (34) has a unique equilibrium point which is globally exponentially stable.

\section{Conclusion}

By using the time scale calculus theory and the Lyapunov functional method, we derive some sufficient conditions to ensure the global exponential stability of learning-based fuzzy networks on time scales. The conditions possess highly important significance and can be easily checked in practice by simple algebraic method; the exponential convergence of the learning dynamics is also considered.

\section{Conflict of Interests}

The authors declare that there is no conflict of interests regarding the publication of this paper.

\section{Acknowledgments}

This research was supported by the National Natural Science Foundation of China under Grants 11101187 and 11361010, the Foundation for Young Professors of Jimei University, the Excellent Youth Foundation of Fujian Province under Grant 2012J06001, NCETFJ under Grant JA11144, and the Foundation of Fujian Higher Education under Grants JA10184 and JA11154.

\section{References}

[1] S. Mohamad, "Global exponential stability in continuoustime and discrete-time delayed bidirectional neural networks," Physica D, vol. 159, no. 3-4, pp. 233-251, 2001. 
[2] T. Yang and L. Yang, "The global stability of fuzzy cellular neural network," IEEE Transactions on Circuits and Systems. I, vol. 43, no. 10, pp. 880-883, 1996.

[3] M. Bohner, "Some oscillation criteria for first order delay dynamic equations," Far East Journal of Applied Mathematics, vol. 18, no. 3, pp. 289-304, 2005.

[4] K. Yuan, J. Cao, and J. Deng, "Exponential stability and periodic solutions of fuzzy cellular neural networks with time-varying delays," Neurocomputing, vol. 69, no. 13-15, pp. 1619-1627, 2006.

[5] T. Yang and L. Yang, "Fuzzy cellular neural network: a new paradigm for image processing," International Journal of Circuit Theory and Applications, vol. 25, no. 6, pp. 459-481, 1997.

[6] X. Yang, X. Liu, Q. Zhang, and P. Liu, "Stability analysis for discrete T-S fuzzy systems," International Journal of Information \& Systems Sciences, vol. 1, no. 3-4, pp. 339-346, 2005.

[7] Y. Liu and W. Tang, "Exponential stability of fuzzy cellular neural networks with constant and time-varying delays," Physics Letters A, vol. 323, no. 3-4, pp. 224-233, 2004.

[8] T. Huang and L. Zhang, "Exponential stability of fuzzy cellular neural networks with unbounded delay," in Proceedings of the Advances in Neural Networks (ISNN '05), vol. 3496 of Lecture Notes in Computer Science, pp. 168-173, 2005.

[9] T. Huang, "Exponential stability of delayed fuzzy cellular neural networks with diffusion," Chaos, Solitons \& Fractals, vol. 31, no. 3, pp. 658-664, 2007.

[10] T. Huang, "Exponential stability of fuzzy cellular neural networks with distributed delay," Physics Letters A, vol. 351, no. 1-2, pp. 48-52, 2006.

[11] Y. Chen and X. Liao, "Novel exponential stability criteria for fuzzy cellular neural networks with time varying delay," in Proceedings of the Advances in Neural Networks (ISNN '04), vol. 3173 of Lecture Notes in Computer Science, pp. 120-125, 2004.

[12] A. Chen and D. Du, "Global exponential stability of delayed BAM network on time scale," Neurocomputing, vol. 71, no. 16-18, pp. 3582-3588, 2008.

[13] Y. Li, X. Chen, and L. Zhao, "Stability and existence of periodic solutions to delayed Cohen-Grossberg BAM neural networks with impulses on time scales," Neurocomputing, vol. 72, no. 7-9, pp. 1621-1630, 2009.

[14] Z. Zhang and K. Liu, "Existence and global exponential stability of a periodic solution to interval general bidirectional associative memory (BAM) neural networks with multiple delays on time scales," Neural Networks, vol. 24, no. 5, pp. 427-439, 2011.

[15] Z. Huang, Y. Raffoul, and C. Cheng, "Scale-limited activating sets and multiperiodicity for threshold-linear networks on time scales," IEEE Transactions on Cybernetics, vol. 44, no. 4, pp. 488499, 2014.

[16] K. Gopalsamy, "Learning dynamics and stability in networks with fuzzy synapses," Dynamic Systems and Applications, vol. 15, no. 3-4, pp. 657-670, 2006.

[17] D. O. Hebb, The Organization of Behaviour, Wiley, New York, NY, USA, 1949.

[18] S. Amari, "Competitive and cooperative aspects in dynamics of neural excitation and self-organization," in Competition and Cooperation in Neural Nets, vol. 45 of Lecture Notes in Biomathematics, pp. 1-28, Springer, Berlin, Germany, 1982.

[19] S. Hilger, "Analysis on measure chains-a unified approach to continuous and discrete calculus," Results in Mathematics, vol. 18, no. 1-2, pp. 18-56, 1990.

[20] S. Hilger, "Differential and difference calculus-unified," Nonlinear Analysis. Theory, Methods \& Applications, vol. 30, pp. 26832694, 1997.
[21] R. Agarwal, M. Bohner, D. O’Regan, and A. Peterson, "Dynamic equations on time scales: a survey," Journal of Computational and Applied Mathematics, vol. 141, no. 1-2, pp. 1-26, 2002.

[22] M. Bohner and A. Peterson, Dynamic Equations on Time Scales: An Introduction with Applications, Birkhäuser, Boston, Mass, USA, 2001.

[23] M. Bohner and A. Peterson, Advances in Dynamic Equations on Time Scales, Birkhäuser, Boston, Mass, USA, 2003.

[24] H. Lu and Z. He, "Global exponential stability of delayed competitive neural networks with different time scales," Neural Networks, vol. 18, no. 3, pp. 243-250, 2005. 


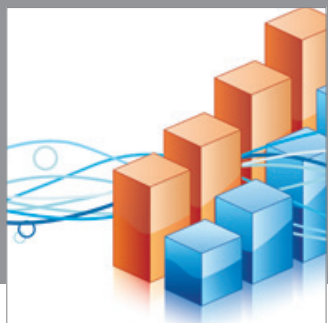

Advances in

Operations Research

mansans

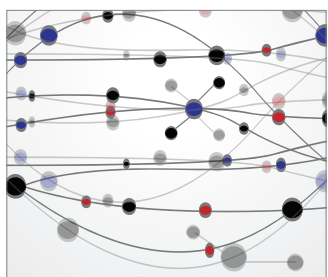

The Scientific World Journal
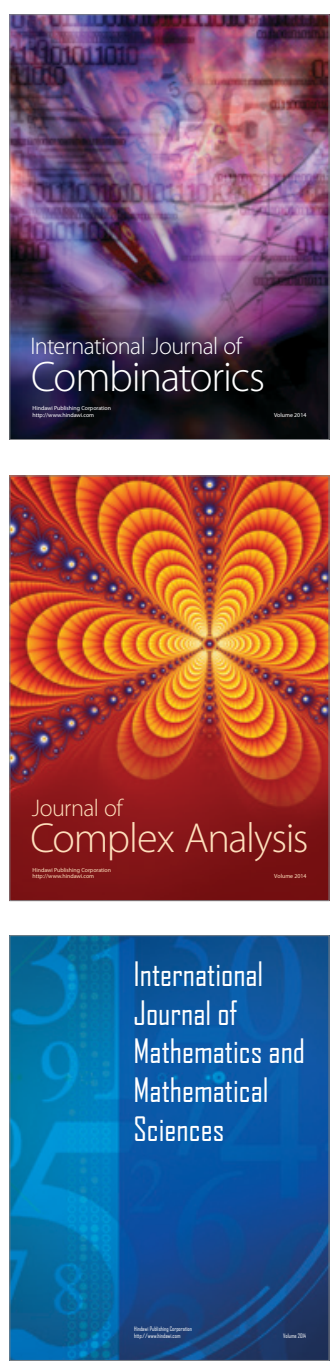
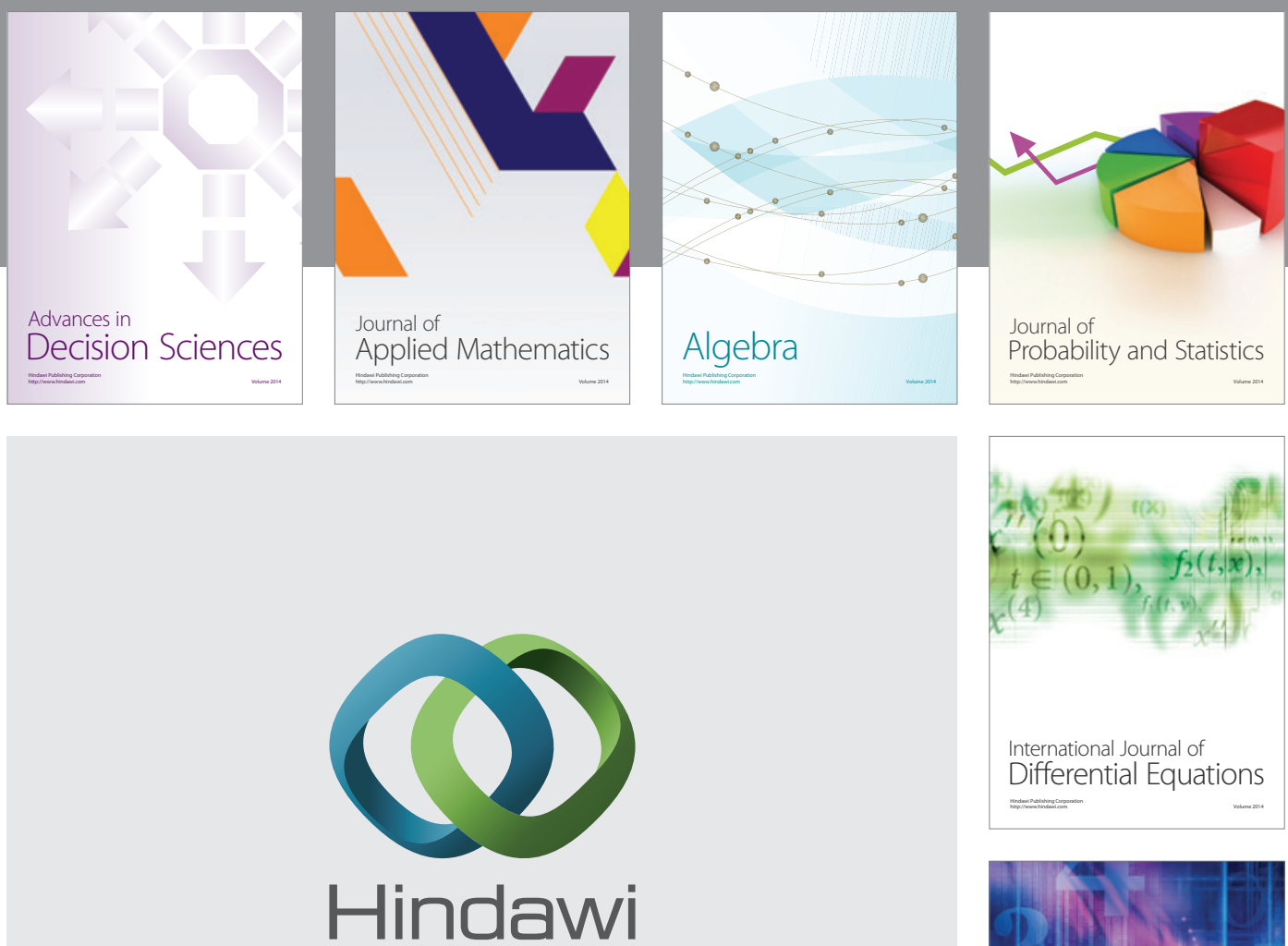

Submit your manuscripts at http://www.hindawi.com
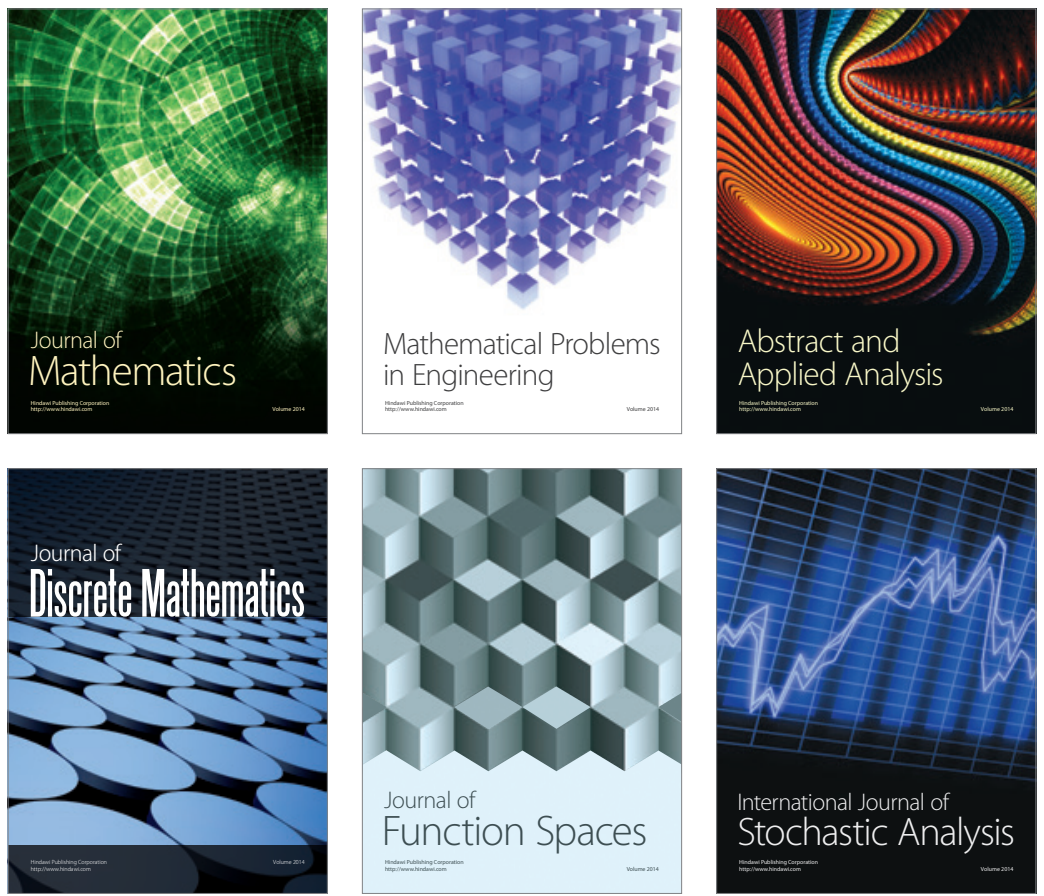

Journal of

Function Spaces

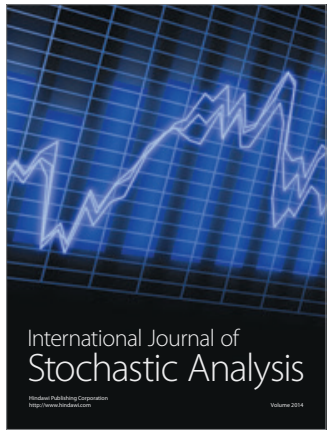

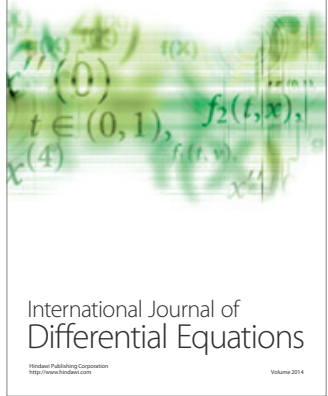
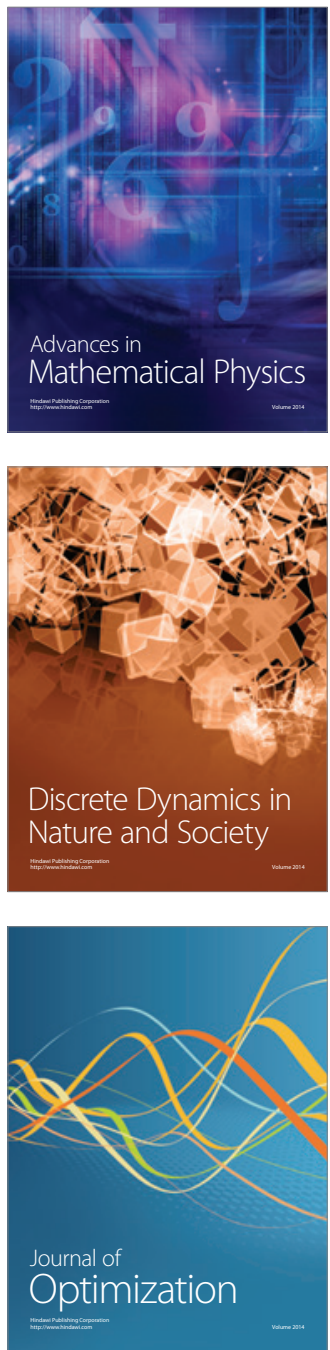